\title{
Variability analysis of 'Persian' acid lime tree selections using agronomic and molecular markers
}

\author{
M.G. Santos ${ }^{1}$, O.S. Passos ${ }^{2}$, W.S. Soares Filho ${ }^{2}$, E.A. Girardi², \\ A.S. Gesteira ${ }^{2}$ and C.F. Ferreira ${ }^{2}$ \\ ${ }^{1}$ Universidade Federal do Recôncavo da Bahia, Cruz das Almas, BA, Brasil \\ ${ }^{2}$ Embrapa Mandioca e Fruticultura, Plant Breeding Department, NUGENE, \\ Cruz das Almas, BA, Brasil \\ Corresponding author: C.F. Ferreira \\ E-mail: claudia.ferreira@embrapa.br
}

Genet. Mol. Res. 12 (4): 4604-4614 (2013)

Received April 5, 2013

Accepted July 18, 2013

Published October 17, 2013

DOI http://dx.doi.org/10.4238/2013.October.17.3

\begin{abstract}
Persian' acid lime (PAL) is the most important triploid commercial citrus crop planted in the world. Little is known about the genetic variability of the selections used in Brazil. Therefore, 25 genotypes originating from the PAL, and three control species, Citrus sunki, C. limon, and C. aurantiifolia, were assessed using intersimple sequence repeat (ISSR) and inter-retrotransposon amplified polymorphism (IRAP) molecular markers and agronomic traits of the fruit. The dendrograms were designed using the mean Euclidean distance for the physicochemical attributes of the fruit (weight, length, diameter, peel color, peel thickness, number of seeds, juice yield, titratable acidity, soluble solids, and ratio) and the Jaccard distances using the data from the ISSR and IRAP molecular markers. In the physicochemical analysis, the genotypes were grouped according to species. The trait that contributed most to the diversity among accessions was the number of seeds. The 17 ISSR primers produced 69 polymorphic bands in the molecular analysis, and the seven IRAP
\end{abstract}


primers generated 30 polymorphic bands. The markers detected polymorphisms within and among the PALs; two groups were formed within the PALs.

Key words: Citrus latifolia; Genetic improvement; Genetic diversity

\section{INTRODUCTION}

The 'Persian' acid lime (PAL) (Citrus latifolia (Yu. Tanaka) Tanaka) is a natural triploid $(2 n=3 x=27)$ that, unlike other acid lime trees such as the 'Galego' acid lime [C. aurantiifolia (Christm.) Swingle], rarely produces seeds. The commercially used genotypes are known as selections, and there are no varieties within the species. These selections have been selected from the rare seeds or buds of superior plants in commercial orchards. However, there are doubts regarding the genetic identity of these plants, which are considered as nucellar, hybrid, or mutant selections, but exhibit characteristics typical of PAL, which in turn could explain the differences in the productivity and development found in plantations of this species.

The use of DNA markers is an attractive approach for defining the genetic identity of plants because it allows the detection of differences between specimens throughout the genome sequence. Molecular markers are powerful tools that have been used in citrus for different purposes, including hybrid identification (Schäfer et al., 2004), genetic diversity analyses (Barkley et al., 2006), linkage map construction (Sankar and Moore, 2001; Gulsen et al., 2010), and cultivar identification (Biswas et al., 2010). Further understanding of the effectiveness of different molecular markers is a key step for characterizing and ranking plant germplasm (Scariot et al., 2007).

The inter-simple sequence repeat (ISSR) and inter-retrotransposon amplified polymorphism (IRAP) markers are notable among the molecular markers that have been used in citrus. The ISSR marker delimits amplified DNA fragments between two microsatellites in opposite directions. This element is a dominant and highly reproducible polymorphic marker that does not require prior knowledge of the DNA sequences for the design of its primers (Bornet and Branchard, 2001).

The IRAP marker amplifies the region between two long terminal repeat (LTR) retrotransposons. This marker exhibits the same characteristics described for the ISSR marker, but requires prior knowledge of the genome for the design of its primers. The LTR regions are highly conserved within families of retrotransposons, enabling their use as markers in several species (Kalendar et al., 1999).

The genetic improvement of PAL is hindered because the data needed to facilitate the collection and selection of superior genotypes are lacking. This study investigated the genetic variability among genotypes originating from PAL seeds using ISSR and IRAP molecular markers and the agronomic traits of the fruit.

\section{MATERIAL AND METHODS}

\section{Plant material}

The genotypes ( 28 in total) used in this study were obtained from the Citrus Active 
Germplasm Bank at Embrapa Mandioca e Fruticultura located in Cruz das Almas, Bahia

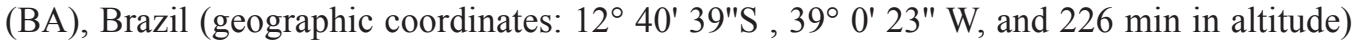
(Table 1). The genotypes were derived from PAL seeds, with the exception of the following species: the 'Sunki' [C. sunki (Hayata) hort. ex. Tanaka] mandarin tree, the 'Fino' [C. limon (L.) Burm.f.] lemon tree, and the 'Galego' (C. aurantiifolia Swingle) acid lime tree, which were used as the controls.

\section{Agronomic characterization of fruit}

A sample of 10 fruits, exhibiting ideal color and firmness for harvest, was randomly selected from around the canopy of each plant. The physicochemical attributes of agronomic relevance were evaluated as follows: the fruit length and diameter were measured using a trough-style ruler $(\mathrm{cm})$; the juice yield was assessed based on the juice mass/fruit mass ratio (\%); the peel thickness was measured using a caliper $(\mathrm{mm})$; the number of seeds per fruit was counted; the total soluble solids (SS) were determined using a direct reading refractometer ( $\left.{ }^{\circ} \mathrm{Brix}\right)$; the titratable acidity (TA), which was expressed in grams of citric acid/100 g juice, was assessed following the dilution of approximately $0.5 \mathrm{~g}$ each juice sample in $40 \mathrm{~mL}$ distilled water and the titration of this solution was assessed with $0.1 \mathrm{~N} \mathrm{NaOH}$ using a digital burette; the ratio maturation index was calculated using the SS/TA ratio; and the peel color was assessed visually according to a rating scale $(1=$ bright green, $2=$ green, $3=$ light green, $4=$ yellow, and 5 = orange-yellow). These evaluations were performed during the second half of 2012.

\section{Characterization using ISSR and IRAP molecular markers}

The genomic DNA was extracted from the young leaves using the cetyltrimethylammonium bromide (CTAB) method (Doyle and Doyle, 1990). The quantity and quality of the DNA were assessed through comparative analysis of the samples on $1 \%$ agarose gels stained with ethidium bromide. The DNA samples were diluted in ultrapure water, and the concentrations were standardized to $5 \mathrm{ng} / \mu \mathrm{L}$.

A total of 24 ISSR and 8 IRAP primers were used. The primer data are shown in Table 2.

The reagent mixture (mix) for the ISSR and IRAP amplification reactions was prepared to a final volume of $15 \mu \mathrm{L}$ and contained the following reagents: $1 \mathrm{X}$ enzyme buffer (50 $\mathrm{mM} \mathrm{KCl}$ and $10 \mathrm{mM}$ Tris-HCl, $\mathrm{pH} 8.3$ ), $1.5 \mathrm{mM} \mathrm{MgCl}_{2}, 0.2 \mathrm{mM}$ dNTPs (dATP, dTTP, dGTP, and $\mathrm{dCTP}$ ), $0.4 \mu \mathrm{M}$ of each primer, $20 \mathrm{ng}$ genomic DNA, and $1 \mathrm{U}$ Taq DNA polymerase (Invitrogen, São Paulo, SP, Brazil).

The amplifications were performed on an Applied Biosystems Veriti ${ }^{\circledR} 96$ Wells thermal cycler using the following amplification program: an initial step at $94^{\circ} \mathrm{C}$ for 3 min followed by 35 cycles of $94^{\circ} \mathrm{C}$ for $40 \mathrm{~s}, 40 \mathrm{~s}$ at an annealing temperature ranging from $48^{\circ}$ to $60^{\circ} \mathrm{C}$ (depending on the primer), and $72^{\circ} \mathrm{C}$ for $1 \mathrm{~min}$, followed by a final extension step at $72^{\circ} \mathrm{C}$ for $5 \mathrm{~min}$. The amplification products were separated by electrophoresis on $3.0 \%$ agarose gels, stained with ethidium bromide, and photographed using the image capture system (Vilber Lourmat). 


\section{Data analysis}

The data from the mean physicochemical attributes of agronomic relevance were used to design a dissimilarity matrix calculated from the mean Euclidian distance. The relative contribution of each attribute to the diversity among genotypes was assessed using the method proposed by Singh (1981) and the GENES software (Cruz, 2006).

The DNA fragments from the ISSR and IRAP reactions were evaluated for the absence ( 0 ) and presence (1) of bands. The matrices of genetic dissimilarity between genotypes were designed based on these data and calculated using the Jaccard coefficient (complement of the Jaccard index $=1-\mathrm{c}$ ) and the GENES software program. In total, three matrices were designed, the first resulting from the ISSR data, the second from the IRAP data, and the third from the combined analysis of these two markers. The genotype-clustering test was performed using the unweighted pair-group method with arithmetic mean (UPGMA) method and the Statistica software (Statistica, 2002). The matrix mean was the criterion adopted for group formation within clusters.

\section{RESULTS AND DISCUSSION}

The clustering shown in Figure 1 was derived from the mean physicochemical attributes (Table 1).

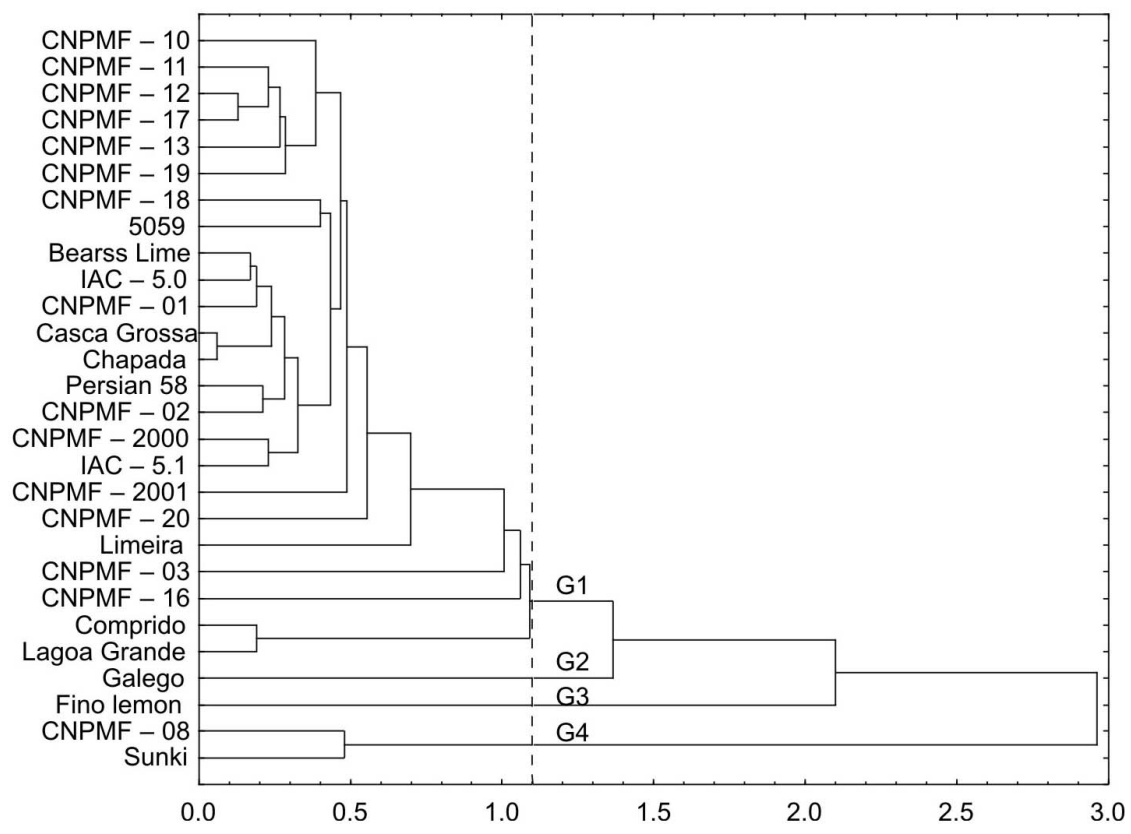

Figure 1. Genetic dissimilarity between 28 citrus genotypes. The dendrogram was designed using the UPGMA clustering method based on the dissimilarity matrix containing 9 quantitative variables of the fruits. 
Table 1. Length (FL), diameter (FD), peel color (PC), peel thickness (PT), seed number (SN), juice yield (JY), titratable acidity (TA), soluble solids (SS) and ratio (RT) of citrus fruit genotypes.

\begin{tabular}{|c|c|c|c|c|c|c|c|c|c|c|}
\hline Type of fruit & Genotypes & FL & FD & $\mathrm{PC}$ & PT & SN & JY & TA & SS & RT \\
\hline Acid lime & Galego & 4.50 & 4.70 & 4.00 & 1.80 & 5.80 & 0.45 & 6.87 & 6.80 & 1.01 \\
\hline Lemon & Fino & 7.21 & 6.00 & 4.00 & 6.20 & 19.0 & 0.35 & 5.56 & 6.60 & 1.19 \\
\hline Lemon & CNPMF - 03 & 7.19 & 6.17 & 3.00 & 5.58 & 0.08 & 0.33 & 5.46 & 7.20 & 1.34 \\
\hline Acid lime & 'Persian' ${ }^{1}$ CNPMF-10 & 6.15 & 6.23 & 2.25 & 3.28 & 1.10 & 0.37 & 6.75 & 7.50 & 1.11 \\
\hline Acid lime & 'Persian' ${ }^{1}$ CNPMF-11 & 6.05 & 6.08 & 2.36 & 4.04 & 0.95 & 0.39 & 6.61 & 7.80 & 1.18 \\
\hline Acid lime & 'Persian' ${ }^{1}$ CNPMF-12 & 5.97 & 6.24 & 2.38 & 3.71 & 1.68 & 0.40 & 6.41 & 8.28 & 1.30 \\
\hline Acid lime & 'Persian' ${ }^{1}$ CNPMF-13 & 5.74 & 5.84 & 2.40 & 3.91 & 0.86 & 0.41 & 6.41 & 8.40 & 1.31 \\
\hline Acid lime & 'Persian' ${ }^{1}$ CNPMF-16 & 5.92 & 6.80 & 2.52 & 3.60 & 3.80 & 0.45 & 5.88 & 9.40 & 1.60 \\
\hline Acid lime & 'Persian' ${ }^{1}$ CNPMF-17 & 5.92 & 6.10 & 2.37 & 3.60 & 1.56 & 0.40 & 6.54 & 8.05 & 1.24 \\
\hline Acid lime & 'Persian' ${ }^{1}$ CNPMF-18 & 5.50 & 5.55 & 2.33 & 3.71 & 1.05 & 0.41 & 7.18 & 7.00 & 0.97 \\
\hline Acid lime & 'Persian' ${ }^{1}$ CNPMF-19 & 5.90 & 6.02 & 2.38 & 2.97 & 1.51 & 0.41 & 6.55 & 8.00 & 1.22 \\
\hline Acid lime & 'Persian' ${ }^{1}$ CNPMF-20 & 5.60 & 5.65 & 2.33 & 4.02 & 3.50 & 0.45 & 6.54 & 8.05 & 1.24 \\
\hline \multirow[t]{2}{*}{ Acid lime } & 'Persian' ${ }^{1}$ CNPMF-2000 & 5.73 & 5.44 & 2.36 & 3.74 & 0.71 & 0.42 & 6.53 & 7.58 & 1.16 \\
\hline & Mean & 5.85 & 5.99 & 2.37 & 3.66 & 1.67 & 0.41 & 6.54 & 8.01 & 1.23 \\
\hline Acid lime & 'Persian' 5059 & 6.33 & 5.69 & 2.20 & 3.21 & 0.05 & 0.44 & 6.87 & 7.44 & 1.08 \\
\hline Acid lime & 'Persian' Bearss Lime & 6.14 & 5.58 & 2.80 & 3.05 & 0.00 & 0.38 & 6.73 & 7.48 & 1.11 \\
\hline Acid lime & 'Persian' CascaGrossa & 5.92 & 5.44 & 2.63 & 3.06 & 0.40 & 0.37 & 6.25 & 7.64 & 1.14 \\
\hline Acid lime & 'Persian' Chapada & 5.89 & 5.43 & 2.61 & 3.06 & 0.95 & 0.37 & 6.21 & 7.66 & 1.12 \\
\hline Acid lime & 'Persian' CNPMF-01 & 6.23 & 5.73 & 2.50 & 3.33 & 0.19 & 0.38 & 6.52 & 7.58 & 1.17 \\
\hline Acid lime & 'Persian' CNPMF-02 & 6.00 & 5.51 & 2.20 & 3.35 & 0.22 & 0.37 & 6.52 & 7.28 & 1.12 \\
\hline Acid lime & 'Persian' CNPMF-2001 & 6.46 & 5.72 & 2.36 & 4.35 & 0.59 & 0.36 & 6.09 & 7.65 & 1.25 \\
\hline Acid lime & 'Persian' Comprido & 6.84 & 6.08 & 2.00 & 0.34 & 1.40 & 0.39 & 6.17 & 7.00 & 1.13 \\
\hline Acid lime & 'Persian' IAC-5.0 & 6.22 & 5.66 & 2.80 & 3.08 & 0.36 & 0.38 & 6.34 & 7.32 & 1.16 \\
\hline Acid lime & 'Persian' IAC-5.1 & 5.81 & 5.58 & 2.50 & 3.57 & 0.43 & 0.39 & 6.84 & 7.68 & 1.12 \\
\hline Acid lime & 'Persian' Lagoagrande & 7.00 & 6.20 & 2.00 & 0.30 & 0.00 & 0.38 & 6.36 & 7.20 & 1.13 \\
\hline Acid lime & 'Persian' Limeira & 6.92 & 6.40 & 2.20 & 3.60 & 0.20 & 0.46 & 5.97 & 7.20 & 1.20 \\
\hline \multirow{2}{*}{ Acid lime } & 'Persian' 58 & 6.42 & 5.60 & 2.20 & 3.04 & 0.06 & 0.39 & 6.64 & 7.36 & 1.11 \\
\hline & Mean & 6.32 & 5.74 & 2.39 & 2.87 & 0.37 & 0.39 & 6.42 & 7.42 & 1.14 \\
\hline Mandarin & CNPMF-08 & 2.90 & 3.30 & 5.00 & 2.60 & 6.00 & 0.19 & 3.50 & 9.70 & 2.77 \\
\hline Mandarin & Sunki & 3.08 & 3.90 & 5.00 & 2.80 & 4.00 & 0.18 & 3.32 & 10.30 & 3.10 \\
\hline
\end{tabular}

'Persian' acid lime with flattened fruits (genotypes in which the female parent plant is 'CNPMF-2000). Cruz das Almas, BA, 2012.

The matrix mean that resulted in this clustering was 1.10. The 'Chapada' and 'Casca Grossa' accessions were the closest, exhibiting 0.06 dissimilarity. In turn, the 'Sunki' mandarin tree and the 'Fino' lemon tree were the most distant, exhibiting 3.35 dissimilarity. The first group (G1) consisted of PALs and 'CNPMF-03', the second (G2) group consisted of the 'Galego' acid lime tree, the third (G3) group consisted of the 'Fino' lemon tree, and the fourth (G4) group consisted of the 'Sunki' mandarin tree and 'CNPMF-08'. The results indicated that the citrus genotypes could be separated by species based on the fruit attributes of a quantitative nature.

Table 1 shows the occurrence of three fruit types: acid lime, lemon, and mandarin. The PALs bore fruit with two distinct shapes, a flattened spheroid with its length shorter than its diameter and an oblong spheroid with its length longer than its diameter. The 'CNPMF-03' and 'CNPMF-08' genotypes, although bearing typical lemon and 'Sunki' mandarin fruits, respectively, were clustered into the same groups because the fruit type was not a variable that was used in the dissimilarity matrix design (Table 1).

According to the Singh (1981) test, the attribute that contributed most to the interspecimen divergence was the mean number of seeds per fruit $(72.5 \%)$. The seed number also contributed significantly to the differentiation of selections of the 'Pera' sweet orange (Citrus sinensis Osbeck) and other varieties of that species (Domingues et al., 2004). 
The PALs with oblong fruits had an average of one seed for every three fruits. The PALs with flattened fruits produced more than one seed per fruit, with the exception of 'CNPMF-11', 'CNPMF-13', and 'CNPMF-2000'. These plants were located near other citrus species, i.e., where there was plenty of pollen, which may explain the occurrence of seeds in the fruits. This situation is unlikely to occur in a commercial orchard because PAL flowers usually fail to produce pollen (Table 1).

In the ISSR analyses, 17 of the 24 primers tested were polymorphic. In total, 125 bands were obtained, 69 of which were polymorphic and 56 of which were monomorphic. The most polymorphic primers were DiCA3'RG, TriATC3'RC, and TriCAA3'RC, each containing six polymorphic bands (Table 2 ).

Table 2. ISSR and IRAP primers used for citrus amplification and their respective sequence, annealing temperature (Ta), monomorphic band number (MBN), polymorphic band number (PBN), and total band number (TBN).

\begin{tabular}{|c|c|c|c|c|c|}
\hline Primer (ISSR) & Sequence & $\mathrm{Ta}\left({ }^{\circ} \mathrm{C}\right)$ & $\mathrm{MBN}$ & PBN & TBN \\
\hline 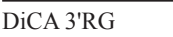 & CACACACACACACACAR $^{1} \mathrm{G}$ & 50 & 4 & 6 & 10 \\
\hline TriCAC 3'RC & CACCACCACCACCACRC & 52 & 2 & 0 & 2 \\
\hline TriTGT 3'YC & TGTTGTTGTTGTTGTY²C & 48 & 2 & 4 & 6 \\
\hline TriTGT 5'CR & CRTGTTGTTGTTGTTGT & 56 & 2 & 4 & 6 \\
\hline TriAAC 3'RC & AACAACAACAACAACRC & 51 & 2 & 5 & 7 \\
\hline TriAAG 3'RC & AAGAAGAAGAAGAAGRC & 48 & 3 & 3 & 6 \\
\hline TriATC 3'RC & ATCATCATCATCATCRC & 48 & 0 & 6 & 6 \\
\hline TriATG 3'RC & ATGATGATGATGATGRC & 51 & 1 & 5 & 6 \\
\hline TriACG 3'RC & ACGACGACGACGACGRC & 58 & 4 & 2 & 6 \\
\hline TriAGA 3'RC & AGAAGAAGAAGAAGARC & 48 & 0 & 4 & 4 \\
\hline TriTAG 3'RC & TAGTAGTAGTAGTAGRC & 48 & 0 & 2 & 2 \\
\hline TriTTC 3'RC & TTCTTCTTCTTCTTCRC & 48 & 3 & 0 & 3 \\
\hline TriTTG 3'RC & TTGTTGTTGTTGTTGRC & 48 & 2 & 4 & 6 \\
\hline TriTCT 3'RC & TCTTCTTCTTCTTCTRC & 48 & 1 & 4 & 5 \\
\hline TriTCC 3'RC & TCCTCCTCCTCCTCCRC & 48 & 5 & 0 & 5 \\
\hline TriTGA 3'RC & TGATGATGATGATGARC & 48 & 3 & 0 & 3 \\
\hline TriCAA 3'RC & CAACAACAACAACAARC & 48 & 3 & 6 & 9 \\
\hline TriCAT 3'RC & CATCATCATCATCATRC & 48 & 2 & 0 & 2 \\
\hline TriCTT 3'RC & CTTCTTCTTCTTCTTRC & 48 & 1 & 5 & 6 \\
\hline TriCTC 3'RC & CTCCTCCTCCTCCTCRC & 48 & 4 & 1 & 5 \\
\hline TriCCG 3'RC & CCGCCGCCGCCGCCGRC & 48 & 3 & 4 & 7 \\
\hline TriCGC 3'RC & CGCCGCCGCCGCCGCRC & 48 & 1 & 0 & 1 \\
\hline TriGAC 3'RC & GACGACGACGACGACRC & 48 & 6 & 0 & 6 \\
\hline TriGTA 3'RC & GTAGTAGTAGTAGTARC & 56 & 2 & 4 & 6 \\
\hline Total & & & 56 & 69 & 125 \\
\hline$\%$ & & & 44.8 & 55.2 & 100.0 \\
\hline Primer (IRAP) & Sequence & $\mathrm{Ta}\left({ }^{\circ} \mathrm{C}\right)$ & MBN & PBN & TBN \\
\hline IRAP $2(\mathrm{LCB})^{3}$ & GGACCTATTTGCCAATGCT & 55 & 1 & 3 & 4 \\
\hline IRAP $3($ LGC) 3 & CCAATTCCGGAAGGTTCTAGG & 60 & 0 & 1 & 1 \\
\hline IRAP $6(\text { SSCC })^{3}$ & ATCTCCCATTTCCGACCACT & 57 & 4 & 3 & 7 \\
\hline SABRINA $^{4}$ & GCAAGCTTCCGTTTCCGC & 51 & 4 & 7 & 11 \\
\hline STOWAWAY $^{4}$ & GCAAGCTTCCGTTTCCGC & 54 & 1 & 7 & 8 \\
\hline SUKKULA $^{4}$ & GATAGGGTCGCATCTTGGGCGTGAC & 60 & 1 & 5 & 6 \\
\hline NIKITA $^{4}$ & CGCTCCAGCGGTACTGCC & 52 & 7 & 4 & 11 \\
\hline Total & & & 18 & 30 & 48 \\
\hline$\%$ & & & 26.9 & 62.5 & 100.0 \\
\hline
\end{tabular}

${ }^{1} \mathrm{R}=(\mathrm{A}, \mathrm{G}) ;{ }^{2} \mathrm{Y}=(\mathrm{C}, \mathrm{T}) ;{ }^{3}$ Biswas et al., 2010; ${ }^{4}$ Baumel et al., 2002.

Initially, there was a separation between the group of lemon and acid lime trees and the group of mandarin trees. The mean dissimilarity between the 28 specimens analyzed was 0.16 . 
The most distant accessions were 'CNPMF-20' and the 'Sunki' mandarin tree (0.44). No differences were found between the 'CNPMF-10', 'CNPMF-13', 'CNPMF-16', 'CNPMF-17', and 'CNPMF-18' and the 'IAC-5', 'IAC-5.1', 'Persian 58', 'CNPMF-01', 'Lagoa Grande', 'Casca Grossa', 'CNPMF-02', 'Comprido', '5059', 'Bearss lime', and 'CNPMF-2001' genotypes. The inability to differentiate amongst the genotypes using ISSR markers suggests the existence of a narrow genetic base.

The dendrogram designed using the data from the ISSR markers demonstrated the formation of three different groups (Figure 2).

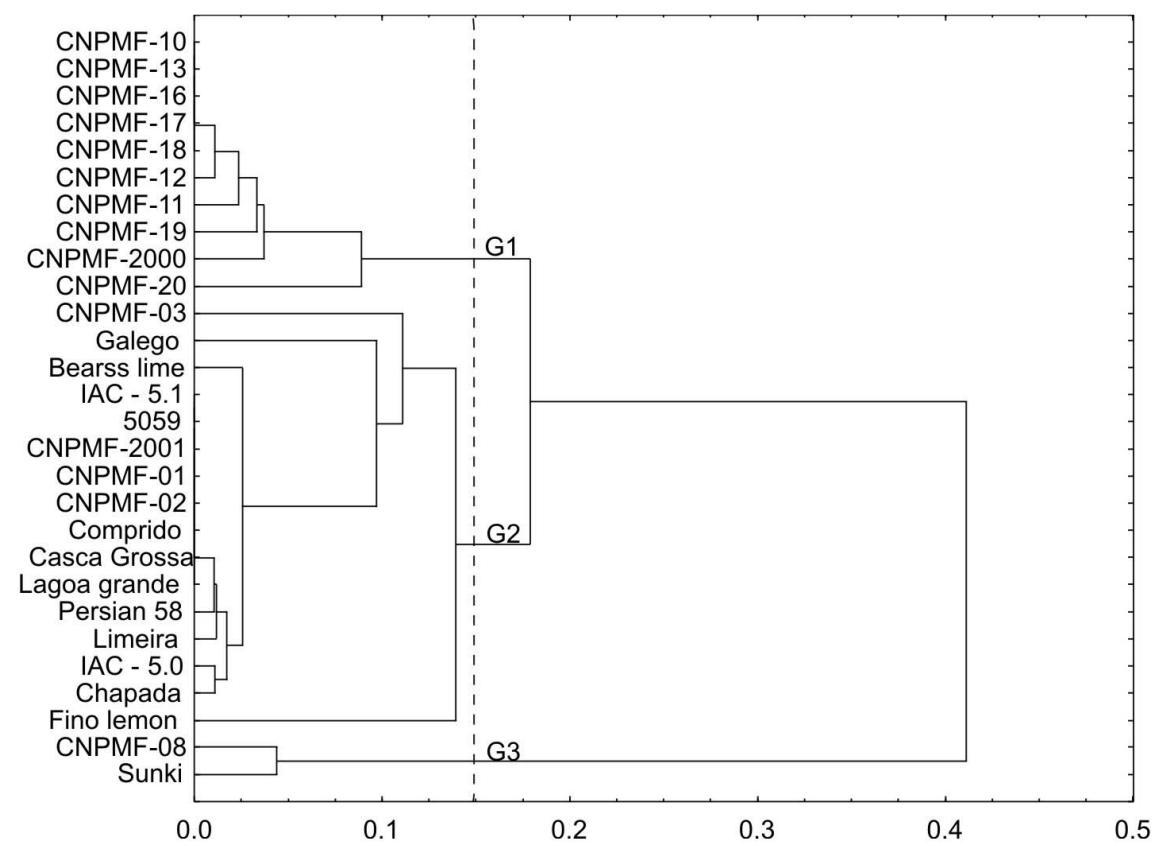

Figure 2. Dendrogram designed for 28 citrus genotypes based on 69 polymorphic bands from the ISSR markers using the UPGMA clustering method.

The PALs with flattened fruits were clustered into group 1. Group 2 consisted of PALs with oblong fruits and the 'CNPMF-03', 'Fino' lemon tree, and 'Galego' acid lime tree genotypes. The third group (G3) consisted of the mandarin trees 'CNPMF-08' and 'Sunki'.

All of the IRAP primers demonstrated polymorphisms; however, the 'Sabrina' and 'Stowaway' varieties exhibited the highest number of polymorphic bands (7), whereas IRAP 3 demonstrated only one polymorphic locus (Table 2). The primers generated 48 bands in total, 30 of which were polymorphic and 18 of which were monomorphic.

The dendrogram designed using the data from the IRAP markers clustered the genotypes into four groups (Figure 3). In contrast to ISSR marker clustering, the 'Fino' lemon tree was separated from the PALs with oblong fruits in a new group (G3).

The most divergent specimens were the 'Sunki' mandarin tree and 'CNPMF-03', with a dissimilarity value of 0.49 . 


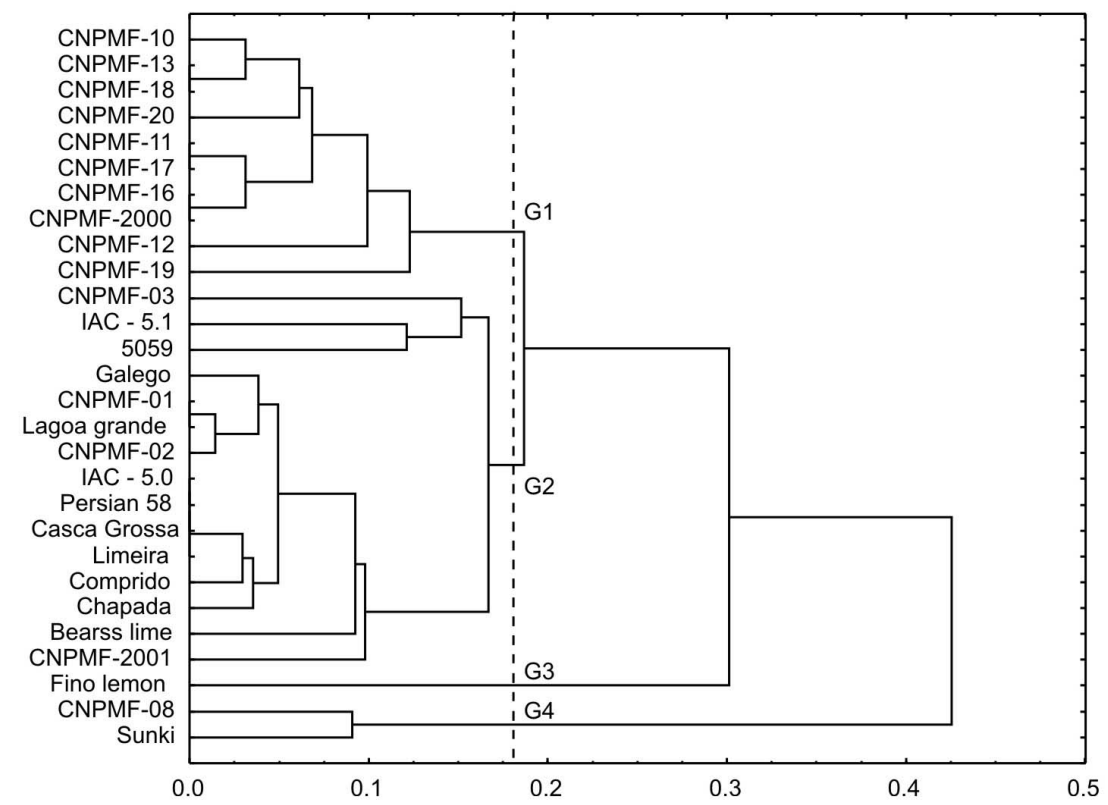

Figure 3. Dendrogram designed for 28 citrus genotypes based on 30 polymorphic bands from the IRAP markers using the UPGMA clustering method.

The genotypes that demonstrated no dissimilarity were 'CNPMF-01', 'CNPMF-02', and 'Lagoa Grande'; 'Persian 58', 'Casca Grossa', 'Limeira', and 'IAC-5'; 'CNPMF-11' and 'CNPMF-17; 'CNPMF-13' and 'CNPMF-18'; and 'CNPMF-16' and 'CNPMF-20'.

The G1 group had the same composition in the IRAP and ISSR dendrograms (Figures 2 and 3). All of the genotypes belonging to G1 originated from PAL 2000 seeds. PAL 2000 was most likely a hybrid of PAL with another male parent plant that was genetically more distant, as single fragments were found in both types of molecular markers for that genotype and its group. Fang et al. (1997) used ISSR markers to analyze citrus varieties such as sweet orange, lemon, and grapefruit trees. The lemon cultivars demonstrated large amounts of single fragments, in contrast to the orange or grapefruit cultivars, indicating the possibility of a polyphyletic origin.

The matrix mean in the combined analysis of the data from the ISSR and IRAP markers was 0.17 , a value close to that found in the analysis using the ISSR markers. The four groups formed in that dendrogram (Figure 4) were the same as those formed using IRAP markers. Similar to the previous group, the most divergent genotypes were the 'Chapada' acid lime and 'Sunki' mandarin trees (0.47).

Many of the PALs were separated by one or both markers; however, the 'CNPMF-01', 'CNPMF-02', and 'Lagoa Grande', and the 'CNPMF-13' and 'CNPMF-18' genotypes did not demonstrate genetic differences, suggesting again that these PAL selections had a narrow genetic base. The failure to detect differences could be explained by the insufficient number of primers used. Furthermore, it would be unwise to dismiss the possibility of differences between these materials because there is evidence of a high rate of somatic mutation in citrus, which would produce closely related specimens (Machado et al., 2005), and molecular markers cannot always detect these differences (Barkley et al., 2006). 


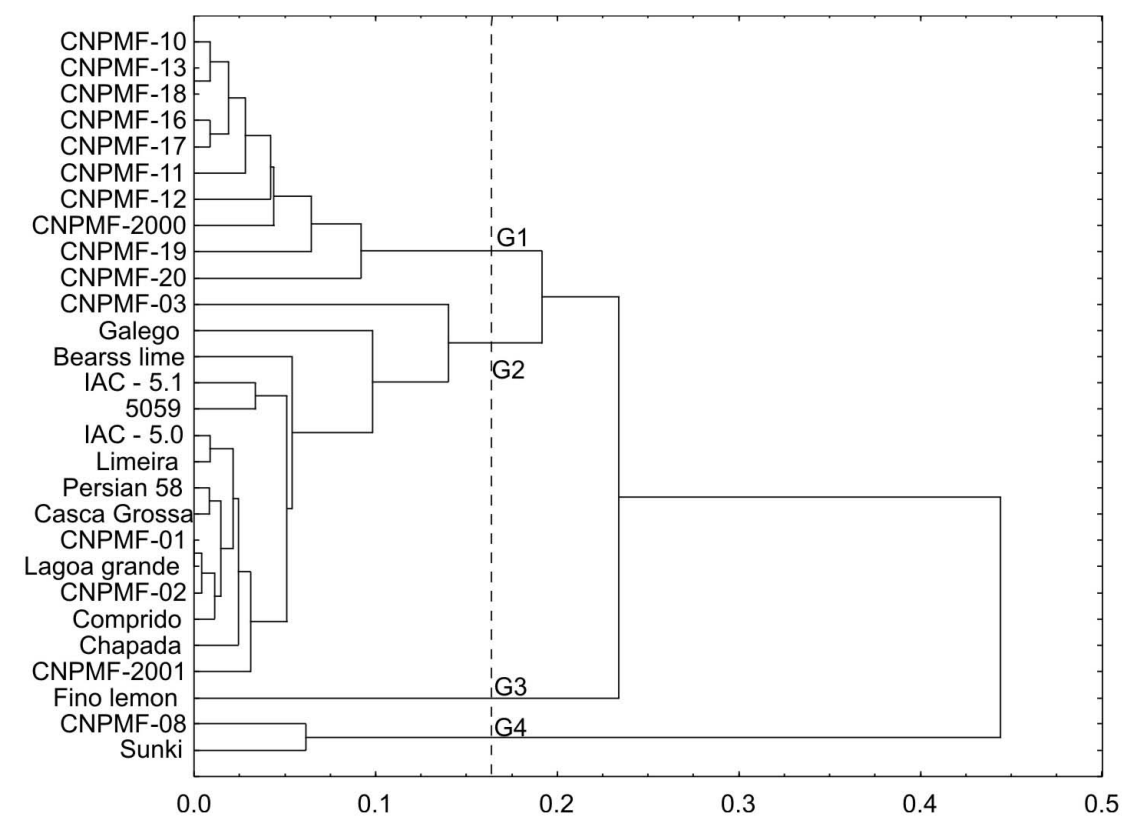

Figure 4. Dendrogram designed based on the dissimilarity matrix containing 69 and 30 polymorphic bands from the ISSR and IRAP markers, respectively, using the UPGMA clustering method.

The ISSR markers demonstrated 55.2\% polymorphic bands, with a mean of 4.06 polymorphic bands per primer, and the IRAP markers demonstrated $62.5 \%$ polymorphic bands, with a mean of 4.28 polymorphic bands per primer (Table 2). Furthermore, the mean dissimilarity of the IRAP matrix (0.19) was higher than that of the ISSR (0.16). The study performed by Bretó et al. (2001) using molecular markers, including ISSRs and IRAPs, to analyze the variability among selections of the 'Clementine' mandarin tree $(C$. clementine hort. ex. Tanaka) corroborates the results of the present study. In the 'Clementine' study, the IRAP markers detected the most polymorphisms (14.6\%), whereas the other markers demonstrated a maximum of $2.4 \%$ polymorphism, highlighting the low variability between the 'Clementine' mandarin tree selections.

Bernet et al. (2004) separated 'Fino', 'Verna', and 'Eureka' lemon trees into a different group from other lemon trees ('Betera', 'Chaparro', 'Librilla', 'Feminello', and 'Dulce') using Tyl-copia class IRAP markers. However, this separation was not observed using Ty3-gypsy class IRAP and ISSR markers, which demonstrated $0 \%$ polymorphisms.

Conversely, Biswas et al. (2010) used ISSR and IRAP markers, among others, to study genetic diversity in Citrus and other related genera, and determined that ISSR and IRAP markers exhibited the same mean number of polymorphic bands per primer (4.67). The ISSR markers detected $84.9 \%$ polymorphisms versus the $82.4 \%$ polymorphisms detected with the IRAPs.

In the combined ISSR and IRAP clustering (Figure 4), the mean value of dissimilarity was 0.13 between PAL and the 'Galego' acid lime tree, 0.18 between the 'Fino' lemon tree and PAL, and 0.24 between the 'Fino' lemon tree and the 'Galego' acid lime tree. This higher 
dissimilarity between the lemon and acid lime trees was also noted by Shahsavar et al. (2007) using ISSR markers to study the phylogenetic relationships between citrus species, clustering PAL with 'Galego' ( $80 \%$ similarity) and detecting lower similarity (approximately 64\%) between the lemon tree and acid lime tree groups. As observed, the use of one type of marker does not exclude using another type of marker since markers can have different annealing regions; therefore, different markers can be used in various situations to detect different levels of polymorphisms, depending on the species.

The genotypes analyzed bore fruits typical of acid lime trees, with the exception of the 'Fino' lemon tree, 'Sunki' mandarin tree, 'CNPMF-08', and 'CNPMF-03' (Table 1). Although 'CNPMF-08' and 'CNPMF-03' were derived from the seeds of PAL fruits, the trees appear to have inherited features that are significantly different from the mother plant. Reece and Childs (1962) also crossbred hybrids of the lime tree that bore fruits exhibiting characteristics similar only to those of the male parent plants. The fruits of 'CNPMF-08' were identical to the 'Sunki' mandarin fruits. These two genotypes were very similar, exhibiting a dissimilarity distance of approximately 0.06 (Figure 4), and almost all of the primers demonstrated fragments specific for these specimens. The 'CNPMF-03' genotype exhibited a number of attributes similar to the lemon tree, which is a vigorous thorny plant that bears ellipsoid fruits, albeit without seeds. The 'CNPMF-03' and 'Fino' lemon tree genotypes demonstrated bands specific for the DiCA 3'RG, TriAAC 3'RC, and TriACG 3'RC primers, although they did not group together. The dissimilarity between these varieties was 0.18 , which was similar to the dissimilarity observed between 'CNPMF-03' and 'Persian' acid lime trees (mean of 0.17). These results indicated that 'CNPMF-08' and 'CNPMF-03' are hybrids of PAL and the 'Sunki' tree and of PAL and the lemon tree, respectively. However, this study failed to explain why interspecific hybrids resulting from crossbreeding using PAL as the female parent plant often carry phenotypic traits that are exclusive to the male parent plant.

\section{ACKNOWLEDGMENTS}

The authors would like to thank Empresa Brasileira de Pesquisa Agropecuária (EMBRAPA) for providing the infrastructure to perform the study.

\section{REFERENCES}

Barkley NA, Roose ML, Krueger RR and Federici CT (2006). Assessing genetic diversity and population structure in a citrus germplasm collection utilizing simple sequence repeat markers (SSRs). Theor. Appl. Genet. 112: 1519-1531.

Baumel A, Ainouche M, Kalendar R and Schulman AH (2002). Retrotransposons and genomic stability in populations of the young allopolyploid species Spartina anglica C.E. Hubbard (Poaceae). Mol. Biol. Evol. 19: 1218-1227.

Bernet GP, Mestre PF, Pina JA and Asíns MJ (2004). Molecular discrimination of lemon cultivars. HortScience 39: 165169.

Biswas MK, Xu Q and Deng X (2010). Utility of RAPD, ISSR, IRAP and REMAP markers for the genetic analysis of Citrus spp. Sci. Hortic. 124: 254-261.

Bornet B and Branchard M (2001). Nonanchored inter simple sequence repeat (ISSR) markers: reproducible and specific tools for genome fingerprinting. Plant Mol. Biol. Rep. 19: 209-215.

Bretó MP, Ruiz C, Pina JA and Asins MJ (2001). The diversification of Citrus clementina Hort. ex Tan., a vegetatively propagated crop species. Mol. Phylogenet. Evol. 21: 285-293.

Cruz CD (2006). Programa Genes: Biometria. Editora UFV, Viçosa.

Domingues ET, Teófilo Sobrinho J, Pompeu Júnior J, Figueiredo JO, et al. (2004). Caracterização de onze clones de laranja 'pera' e seis variedades assemelhadas. Laranja 25: 111-138. 
Doyle JJ and Doyle JL (1990). Isolation of plant DNA from fresh tissue. Focus Rockville 12: 13-15.

Fang DQ, Roose ML, Krueger RR and Federici CT (1997). Fingerprinting trifoliate orange germplasm accessions with isozymes, RFLPs and inter-simple sequence repeat markers. Theor. Appl. Genet. 95: 211-219.

Gulsen O, Uzun A, Canan I, Seday U, et al. (2010). A new citrus linkage map based on SRAP, SSR, ISSR, POGP, RGA and RAPD markers. Euphytica 173: 265-277.

Kalendar R, Grob T, Regina MT, Suoniemi A, et al. (1999). IRAP and REMAP: two new retrotransposon-based DNA fingerprinting techniques. Theor. Appl. Genet. 98: 704-711.

Machado MA, Cristofani C, Amaral AM and Oliveira AC (2005). Genética, Melhoramento e Biotecnologia de Citros. In: Citros (Mattos Junior D, Negri JD, Pio RM and Pompeu Junior J, eds.). Instituto Agronômico e Fundag, Campinas, 221-277.

Reece PC and Childs JFL (1962). Character differences among seedlings of the Persian lime. Florida State Hortic. Soc. 75: 110-116.

Sankar AA and Moore GA (2001). Evaluation of inter-simple sequence repeat analysis for mapping in Citrus and extension of the genetic linkage map. Theor. Appl. Genet. 102: 206-214.

Scariot V, De Keyser E, Handa T and De Riek J (2007). Comparative study of the discriminating capacity and effectiveness of AFLP, STMS and EST markers in assessing genetic relationships among evergreen azaleas. Plant Breed. 126: 207-212.

Schäfer G, Bastianel M and Dornelles ALC (2004). Identificação de plântulas zigóticas de trifoliata com o uso de marcadores moleculares RAPD. Pesq. Agropec. Bras. 39: 167-172.

Shahsavar AR, Izadpanah K, Tafazoli E and Sayed Tabatabaei BE (2007). Characterization of citrus germplasm including unknown variants by inter-simple sequence repeat (ISSR) markers. Sci. Hortic. 112: 310-314.

Singh D (1981). The relative importance of characters affecting genetic divergence. Indian J. Genet. Plant Breed. 41: $237-245$.

Statistica (2002). STATISTICA for Windows v. 6.0: Computer Program Manual. Editora StatSoft Inc. (CD-Rom), Tulsa. 